\title{
Neonatal diabetes mellitus due to a novel variant in the INS gene
}

\author{
Sarah E. Laurenzano, ${ }^{1}$ Cory McFall, ${ }^{2}$ Linda Nguyen, ${ }^{3}$ Dipal Savla, ${ }^{3}$ \\ Nicole G. Coufal, ${ }^{2}$ Meredith S. Wright, ${ }^{4}$ Mari Tokita, ${ }^{4}$ David Dimmock, ${ }^{4}$ \\ Stephen F. Kingsmore, ${ }^{4}$ and Ron S. Newfield ${ }^{1}$
}

${ }^{1}$ Division of Pediatric Endocrinology, Department of Pediatrics, University of California, San Diego, La Jolla, California 92093, USA; ${ }^{2}$ Division of Pediatric Intensive Care Medicine, Department of Pediatrics, University of California, San Diego, La Jolla, California 92093, USA; ${ }^{3}$ Department of Pediatrics, University of California, San Diego, La Jolla, California 92093, USA; ${ }^{4}$ Rady Children's Institute for Genomic Medicine, San Diego, California 92123, USA

\begin{abstract}
Neonatal diabetes mellitus (NDM) is a rare condition that presents with diabetes in the first few months of life. The treatment of NDM may differ depending on the genetic etiology, with numerous studies showing the benefit of sulfonylurea therapy in cases caused by mutations in KCNJ11 or ABCC8. Mutations in the insulin gene (INS) have also been identified as causes of NDM; these cases are generally best treated with insulin alone. We report a case of a female infant born small for gestational age (SGA) at late preterm diagnosed with NDM at 7 wk of life who was found by rapid whole-genome sequencing to harbor a novel de novo c. $26 \mathrm{C}>\mathrm{G}$ (p.Pro9Arg) variant in the INS gene. She presented with diabetic ketoacidosis, which responded to insulin therapy. She did not respond to empiric trial of sulfonylurea therapy early in her hospital course, and it was discontinued once a genetic diagnosis was made. Early genetic evaluation in patients presenting with NDM is essential to optimize therapeutic decision-making.
\end{abstract}

Corresponding author: rnewfield@rchsd.org

(c) 2019 Laurenzano et al. This article is distributed under the terms of the Creative Commons Attribution-NonCommercial License, which permits reuse and redistribution, except for commercial purposes, provided that the original author and source are credited.

Ontology terms: diabetes mellitus; diabetic ketoacidosis

Published by Cold Spring Harbor Laboratory Press

doi:10.1101/mcs.a004085

\section{INTRODUCTION}

Neonatal diabetes mellitus (NDM) is a rare disorder that is defined by the onset of diabetes before age 6 mo. Its incidence has been estimated at 1 in every 90,000-400,000 births (Polak and Cave 2007; lafusco and Massa 2012). Infants typically present with small size for gestational age (SGA), dehydration, failure to thrive, and hyperglycemia with or without ketonuria or ketoacidosis. NDM can be either permanent or transient and is likely to have an underlying genetic cause. The most common genetic etiologies of NDM in children born to nonconsanguineous parents include mutations in $K C N J 11$ or $A B C C 8$, the genes encoding the potassium ATP-sensitive channel subunits Kir6.2 and SUR1, respectively; mutations involving the insulin (INS) gene; or methylation abnormalities of Chromosome 6q24 (De Franco et al. 2015). Both autosomal recessive and autosomal dominant INS mutations have been described. Patients with defects in KCNJ11 or ABCC8 can often be managed well with sulfonylurea therapy (Babenko et al. 2006; Pearson et al. 2006). In cases involving other genetic mutations, insulin remains the mainstay of treatment. Here, we present a case of NDM caused by a de novo variant in the INS gene that highlights the utility of rapid whole-genome sequencing (WGS) in the clinical care of NDM. 


\section{CASE PRESENTATION}

The patient is a female born at $36 \mathrm{wk}$ and $5 \mathrm{~d}$ gestation via Cesarean section because of placenta previa. The history was notable for classification of the infant as SGA by weight (birth weight of $2.01 \mathrm{~kg},<1$ st percentile) and for report of enlarged kidneys on prenatal ultrasound. The postnatal history included a 4-d NICU stay, with initial respiratory distress (resolved by discharge) and sepsis evaluation; note was also made of transient hyperglycemia presumed due to an exaggerated stress response, which also resolved. In the outpatient setting, she was followed by her pediatrician for poor weight gain. She subsequently presented at the age of $7 \mathrm{wk}$ to an urgent care facility with tachypnea and a single episode of emesis. She was lethargic, tachycardic, dehydrated, and in obvious respiratory distress. She was transferred to the pediatric emergency department for further care.

On arrival to our facility, the patient was again noted to be in respiratory distress, and her weight was again noted to be low $(2.9 \mathrm{~kg},<1$ st percentile for age). Laboratory studies showed a high anion gap of $22 \mathrm{mmol} / \mathrm{L}$ (reference range $5-20 \mathrm{mmol} / \mathrm{L}$ ), metabolic acidosis (venous $\mathrm{pH} 7.096$ [reference range 7.31-7.41]), serum bicarbonate $(6 \mathrm{mmol} / \mathrm{L}$ [reference range 18-27 mmol/L]), and profound hyperglycemia (initial serum glucose $734 \mathrm{mg} / \mathrm{dL}$ [40.7 mmol/L], reference range $60-110 \mathrm{mg} / \mathrm{dL}$ [3.3-6.1 mmol/L]). Isotonic fluid resuscitation was initiated with a total of $30 \mathrm{~mL} / \mathrm{kg}$ of normal saline, and once serum blood glucose decreased below $600 \mathrm{mg} / \mathrm{dL}(33.3 \mathrm{mmol} / \mathrm{L})$, a regular insulin infusion was begun at 0.05 units $/ \mathrm{kg} / \mathrm{h}$ and soon after was increased to $0.1 \mathrm{units} / \mathrm{kg} / \mathrm{h}$. The infant was admitted to the pediatric intensive care unit (PICU).

During the early portion of her PICU stay, blood glucose was monitored hourly and venous blood gas every $2 \mathrm{~h}$ per protocol. Maintenance i.v. fluids were provided, with the addition of dextrose as appropriate with decreasing blood glucose. Ketoacidosis was deemed resolved within $\sim 8 \mathrm{~h}$ of admission, at which time feeding with breast milk was allowed every 3 $\mathrm{h}$ and subcutaneous insulin started. The initial regimen included low doses of glargine insulin and correctional dilute lispro insulin, the doses of which required frequent adjustment because of widely variable blood glucose values. Additional laboratory results from the PICU stay included a hemoglobin A1c (HbA1c) of 6.3\% (normal range $4.2 \%-5.6 \%$ ), very low C-peptide $(0.16 \mathrm{ng} / \mathrm{mL}$, reference range $0.8-3.1 \mathrm{ng} / \mathrm{mL})$, and low insulin level $(1 \mathrm{mU} / \mathrm{mL}$, reference range $<17 \mathrm{mU} / \mathrm{mL}$ ), which were inappropriately low for her hyperglycemic state, and negative insulin autoantibody (titer $<0.4 \mathrm{U} / \mathrm{mL}$ ). She was nominated for rapid WGS by our facility's genomics institute while in the PICU on hospital day 5 . She was able to be transferred out of the PICU by hospital day 6, and a sample for WGS was collected that day.

Over the next few days, she continued to have widely variable blood glucose levels despite adjustments in dosing and timing of both glargine and dilute lispro insulin. With the knowledge that the majority of neonatal diabetes is caused by mutations in either ABCC 8 or KCNJ11 that may be sulfonylurea-responsive, she was empirically started on twice-daily glyburide on day 7 of admission, which did not improve her hyperglycemia. On hospital day 8 , genomic test results were available and showed a de novo heterozygous c.26C>G (p.Pro9Arg) variant in the INS gene (Table 1).

\begin{tabular}{lcccccccc}
\hline Table 1. Variant table & HGV DNA & $\begin{array}{c}\text { HGVS } \\
\text { protein } \\
\text { reference }\end{array}$ & $\begin{array}{c}\text { Variant } \\
\text { Type }\end{array}$ & $\begin{array}{c}\text { Predicted effect } \\
\text { (substitution, } \\
\text { deletion, etc.) }\end{array}$ & $\begin{array}{c}\text { dbSNP/ } \\
\text { dbVar ID }\end{array}$ & $\begin{array}{c}\text { Genotype } \\
\text { (heterozygous/ } \\
\text { homozygous) }\end{array}$ & ClinVarlD \\
\hline INS & $\begin{array}{c}11: 2182176 \\
\left(N M \_001185098.1\right)\end{array}$ & c.26C>G & p.Pro9Arg & Missense & Likely pathogenic & None & Heterozygous & SCV000898466.1 \\
\hline
\end{tabular}


The p.Pro9Arg variant in INS, which has not been previously reported in the literature, is absent from the Exome Aggregation Consortium (ExAC) and Genome Aggregation (gnomAD) population databases. It involves a highly conserved amino acid (phyloPVertebrate: 3.35 ) and is predicted by multiple in silico tools to have a deleterious effect on protein function (MutationTaster: 0.991; SIFT: 0.006; PolyPhen-2: 0.917). Analysis of the parental samples was negative for the c. $26 \mathrm{C}>\mathrm{G}$ (p.Pro9Arg) variant, indicating that it likely occurred as a de novo event in the patient. Therefore, the variant was classified as likely pathogenic. Glyburide was stopped within $36 \mathrm{~h}$ after it was started based on the molecular diagnosis, which indicated that sulfonylurea therapy would not be an effective adjunct.

In an effort to lessen glycemic variability and decrease the frequency of correctional dilute lispro insulin injections in an infant with multiple daily feeds, intermediate-acting insulin $(\mathrm{NPH})$ was added and insulin glargine was discontinued, allowing the family to give lispro correctional injections less frequently. With dose titration over the next few days, blood glucose values were generally within the acceptable range, although some variability continued. She was able to be discharged on hospital day 12 on a regimen of NPH 1.5 units every $8 \mathrm{~h}(1.55 \mathrm{units} / \mathrm{kg} / \mathrm{d}$ for admission weight of $2.9 \mathrm{~kg})$ and dilute lispro given for hyperglycemia as needed. Recognizing her increased risk for hypoglycemia and glycemic variability because of her very young age and small size, we applied for a continuous glucose sensor (DexCom) during her hospital stay, which the family received within 2 wk of hospital discharge. The family remained in close communication with the endocrinology team following hospital discharge for review of blood glucose values and insulin adjustments as appropriate. At follow-up at age $5 \mathrm{mo}$, her $\mathrm{HbA} 1 \mathrm{c}$ was $6.8 \%$ and weight had improved (weight for length at the 93rd percentile). Her regimen included twice-daily NPH insulin and twice-daily short-acting insulin (total of 0.46 units $/ \mathrm{kg} / \mathrm{d}$ ) for carbohydrate coverage, with correctional insulin given in between those times as needed. At follow-up at age 9 mo, her $\mathrm{HbA} 1 \mathrm{c}$ was $7 \%$ and weight for length was at the 97th percentile. Her regimen was adjusted to discontinue $\mathrm{NPH}$ insulin and reintroduce once-daily insulin glargine along with short-acting insulin with all meals in a typical basal-bolus regimen (total of 0.77 units $/ \mathrm{kg} / \mathrm{d}$ ).

\section{DISCUSSION}

There is significant genetic heterogeneity in NDM, an entity that may be transient or permanent. In permanent neonatal diabetes (PNDM), the most common genetic mutations seen are in $K C N J 11$ and $A B C C 8$, accounting for close to $50 \%$ of PNDM; INS gene mutations account for $\sim 20 \%$ of PNDM, with mutations in other genes (such as GCK and PDX1) occurring less commonly (De León et al. 2008). Both missense and loss-of-function variants in the INS gene have been described in individuals with PNDM (Edghill et al. 2008; Liu et al. 2015), with different mutation types affecting different steps in insulin biosynthesis and function but all producing the phenotype of PNDM. The INS gene is translated into the precursor preproinsulin, which undergoes removal of its signal peptide to generate proinsulin, after which further intracellular modifications including excision of C-peptide result in the mature form of insulin (Støy 2014). The novel p.Pro9Arg missense variant detected in our patient occurs in the signal peptide of the preproinsulin molecule, in an amino acid residue that is highly conserved.

Although it could be hypothesized that the p.Pro9Arg variant disrupts insulin biosynthesis very early in the process, given the heterozygous nature of the mutation in this case, it is difficult to attribute development of overt, early-onset diabetes to insulin haploinsufficiency alone. Indeed, the molecular mechanisms underlying the development of PNDM in the setting of heterozygous INS mutations are not yet completely understood. Increased endoplasmic reticulum (ER) stress, with or without increased $\beta$-cell apoptosis, have been implicated (Oyadomari et al. 2002; Balboa et al. 2018). Recently, Balboa et al. (2018) reported that 
iPSC-derived INS-mutant $\beta$-like cells transplanted into mice increased ER stress, led to defective $\beta$-cell mass expansion, and reduced insulin secretion. Liu et al. $(2010,2015)$, in reviewing the diversity of the MIDY (mutant INS-gene-induced diabetes of youth) phenotype, describe the hypothesis of "gain of toxic function," which is felt to be heavily implicated in the development of diabetes and which may be initiated by abnormal interactions between mutant and wild-type proinsulin molecules in the ER of $\beta$ cells.

Beyond providing information useful in understanding the pathogenesis of NDM in a case such as this, results of rapid genetic sequencing can be key in guiding management. In particular, it is well-known that NDM resulting from mutations in KCNJ11 and ABCC8 may respond well to sulfonylurea (SU) therapy. In general, it is felt that $\mathrm{SU}$ therapy is ineffective in cases of NDM due to INS gene mutations. In our case, SU therapy was empirically started while genetic testing results were pending. When these became available in the span of just $2 \mathrm{~d}$, therapy was able to be appropriately tailored to the patient, as it was evident that SU therapy would be ineffective based on the mechanism of disease. Moreover, although empiric therapy while awaiting genetic results is not uncommon, one case of insulin resistance emerging after addition of SU therapy in a patient with transient NDM due to a mutation in the promoter region of INS has been described (Yildiz et al. 2018). Although adverse effects with adjunctive SU therapy have not been commonly described, and although there may be significant genetic and clinical differences between patients with transient or permanent NDM, we feel that whenever possible it is important to prioritize ancillary genetic testing that may substantially impact management. Although other testing methods (e.g., targeted panel testing, whole-exome sequencing) would also have provided an accurate diagnosis in this case, WGS performed at our facility has a shorter turnaround time than most commercially available gene panel and exome sequencing tests. In fragile patients requiring intensive care, rapid diagnosis is crucial for timely, specific treatment, which results in improved outcomes and decreased length of stay (Farnaes et al. 2018).

A clinical feature of interest in this case was the diagnosis of bilateral kidney enlargement made on prenatal ultrasound. Her postnatal course has been notable for ultrasound during admission for DKA showing right kidney length of $5.5 \mathrm{~cm}$ and left kidney length $6.2 \mathrm{~cm}$ (both high-normal in size [Konuş et al. 1998]) as well as moderate left hydronephrosis, SFU (Society for Fetal Urology) Grade 3. Nephromegaly and/or hydronephrosis are not commonly described in individuals with neonatal diabetes in the available literature; we speculate that this could be related to renal hyperfiltration due to hyperglycemia and polyuria in utero.

An additional clinical feature worth noting in this case includes the $\mathrm{HbA} 1 \mathrm{c}$ of $6.3 \%$ at diagnosis; alternative estimates of glycemic control, such as fructosamine or glycated albumin, were not measured in our patient. It has been described (Suzuki and Koga 2014; Wirth et al. 2018) that despite the widespread clinical use of HbA1c to estimate glycemic control in diabetes mellitus and because of the persistence of high levels of fetal hemoglobin in neonates, $\mathrm{HbA} 1 \mathrm{c}$ is not a reliable measure of glycemic control in NDM. We could have measured fructosamine if the diagnosis of diabetes was in doubt and/or performed hemoglobin subtype analysis to correct the HbA1c (Suzuki and Koga 2014), but there was no clinical justification for the additional testing in light of the clinical presentation and significant ketoacidosis.

When it comes to method of insulin delivery, multiple different regimens of subcutaneous insulin therapy by injection were utilized in this case (including different combinations of long-, intermediate-, and short-acting insulin). Early on, a combination of intermediate- and shortacting insulin (with transition to typical basal-bolus regimen later in infancy) was effective in avoiding hypoglycemia and reducing (although certainly not eliminating) glycemic variability; early availability of a continuous glucose sensor was crucial in allowing for frequent adjustments to the patient's insulin regimen. The successful use of insulin pump therapy (continuous subcutaneous insulin infusion [CSII]) has been well described in cases of NDM (Tubiana-Tufi 
COLD SPRING HARBOR Molecular Case Studies
INS-associated neonatal diabetes mellitus
2007), even being introduced during initial hospital stay in a case of transient NDM (Park et al. 2013). Challenges common to both injection and pump therapy include frequent feedings, limited subcutaneous fat stores, and need for minute doses of insulin in patients with NDM. CSII was discussed with the family, and they felt they would prefer to continue with multiple daily injections for the time being, but strong consideration is being given to introducing CSII in the near future, particularly as her size and total daily insulin dose increase.

In summary, we present a case of neonatal diabetes due to a heterozygous de novo INS variant c.26C $>$ G (p.Pro9Arg), a variant affecting the signal peptide of preproinsulin that has not previously been described in cases of NDM due to INS mutations. The development of diabetes in heterozygous INS mutations has been linked to a potential gain-of-toxic-function mechanism as well as to increased ER stress in the setting of (pre)proinsulin misfolding. From the clinical standpoint, this case highlights the utility of genetic testing with rapid turnaround time in therapeutic decision-making. Practical challenges remain in treating our very youngest diabetic patients to achieve glycemic targets without hypoglycemia, which may be mitigated by immediate and widespread availability of continuous glucose sensors to such patients.

\section{METHODS}

Consent for rapid WGS was obtained for the proband and her parents on hospital day 5. Following DNA extraction from whole blood, sequencing libraries were constructed using the TruSeqDNA PCR-Free Library Prep kit according to the manufacturer's instructions. Paired-end sequencing ( $2 \times 100 \mathrm{bp}$ ) was performed with Illumina chemistry on a NovaSeq 6000 to a mean depth of coverage of greater than $40 \times$ for the patient and greater than $30 \times$ for parental samples (Table 2). The Edico Dragen processor (Illumina) was used for rapid alignment and nucleotide variant calling. Variant analysis and interpretation were performed using the Opal Clinical Interface (Fabric Genomics), which employs proprietary algorithms to prioritize candidate variants by integrating phenotypic and genomic data. Variants were filtered to retain those with allele frequencies of $<0.5 \%$ in multiple population databases including gnomAD, Exome Variant Server, 1000 Genomes, and ExAC. A custom gene panel was then created in Phenolyzer (Yang et al. 2015) and used to prioritize review of variants detected in genes known to be associated with hyperglycemia (HPO: 0003074). Manual curation was performed and the c.26C>G (p.Pro9Arg) variant in the INS gene was classified as likely pathogenic (one strong, one moderate, and one supporting criteria) according to the ACMG/AMP guidelines for the interpretation of sequence variants (Richards et al. 2015). This variant was orthogonally confirmed by Sanger sequencing using the following custom designed forward $(F)$ and reverse $(R)$ primers: F: TTAAAAAAGTGCACCTGACCC CCT and R: TCCAAGGGCCTTTGCGTCAG. Sanger sequencing confirmed the variant was heterozygous in the patient and negative in the parental samples.

\section{ADDITIONAL INFORMATION}

\section{Data Deposition and Access}

The causative variant has been deposited at ClinVar (https://www.ncbi.nih.nlm.gov/clinvar/) with accession number SCV000898466.1. All data associated with this study are present in the paper or are available at the Longitudinal Pediatric Data Resource under a material transfer agreement or data use agreement, as appropriate, and subject to the limitations of the informed consent documents for each subject (Accession Number nbs000003.v1.p; https://www.nbstrn.org/research-tools/longitudinal-pediatric-data-resource). 


\begin{tabular}{|c|c|c|c|c|}
\hline Trio & R18AA922 & R18AA923 & R18AA924 & Units \\
\hline Date sample was run & Jun 42018 & Jun 12018 & Jun 12018 & \\
\hline Sex & $\mathrm{F}$ & $\mathrm{F}$ (mother) & M (father) & \\
\hline Yield: raw/bulk & 179.5 & 116.2 & 125.4 & \\
\hline$\%$ mapped & $98.50 \%$ & $98.50 \%$ & $98.20 \%$ & $\%$ \\
\hline$\%$ duplicates & $7.90 \%$ & $7.10 \%$ & $8.10 \%$ & $\%$ \\
\hline Yield & 176.8 & 114.4 & 123.1 & Gbp \\
\hline Insert size: mean & 423.6 & 413.1 & 407.3 & $\mathrm{bp}$ \\
\hline Average and median coverage across genome & 49.6 & 32.3 & 34.3 & $x$ \\
\hline Average coverage over OMIM genes & 46.7 & 31.0 & 32.6 & $\times$ \\
\hline \# of OMIM genes with coverage at $<10 x$ & 314 & 703 & 977 & \\
\hline$\#$ of OMIM genes with $100 \%$ coverage at $\geq 10 x$ & $97.8 \%$ & $95.0 \%$ & $93.1 \%$ & $\%$ \\
\hline$\#$ of OMIM genes with $100 \%$ coverage at $\geq 20 x$ & $90.0 \%$ & $21.2 \%$ & $35.0 \%$ & $\%$ \\
\hline \# of OMIM genes with $100 \%$ coverage at $\geq 30 \times$ & $37.3 \%$ & $2.3 \%$ & $2.7 \%$ & $\%$ \\
\hline \# of genes with $100 \%$ coverage at $\geq 40 x$ & $4.0 \%$ & $0.5 \%$ & $0.9 \%$ & \\
\hline \multicolumn{5}{|l|}{ Variation (VCF) metrics } \\
\hline \# of calls total & $4,968,231$ & $4,962,138$ & $4,837,935$ & \\
\hline \# of PASS calls & $4,887,184$ & $4,903,387$ & $4,775,895$ & \\
\hline \# of calls total coding & 24,742 & 24,702 & 24,319 & \\
\hline Total \# of SNVs & $3,969,838$ & $4,008,954$ & $3,897,489$ & \\
\hline Total \# of Indels & 917,346 & 894,433 & 878,406 & \\
\hline Hom/Het ratio (in coding regions) & 0.55 & 0.54 & 0.60 & ratio \\
\hline Ti/Tv ratio (in coding regions) & 1.96 & 1.97 & 1.97 & ratio \\
\hline \# of het calls (\# of hom call) & $3,186,900(1,781,331)$ & $3,208,560(1,753,578)$ & $3,007,910(1,830,025)$ & units \\
\hline
\end{tabular}

Competing Interest Statement

The authors have declared no competing interest.

\section{Referees}

Ralph J. DeBerardinis

Anonymous

Received February 22, 2019; accepted in revised form June 3, 2019.

\section{Ethics Statement}

Written research consent obtained from patient's parents. Molecular genetic testing was performed under an IRB-approved research protocol with Dr. Stephen Kingsmore as the PI: "Prenatal Precision Medicine (NSIGHT2): A Randomized, Blinded, Prospective Study of the Clinical Utility of Rapid Genomic Sequencing for Infants in the Acute-care Setting," ClinicalTrials.gov ID: NCT03211039.

\section{Acknowledgments}

We wish to acknowledge the physicians and nurses involved in the patient's clinical care and the Rady Children's Institute of Genomic Medicine.

\section{Author Contributions}

S.E.L. was the primary author and was involved in the patient's clinical care. C M., L.N., and D.S. were involved in the patient's clinical care and in writing and review of the manuscript. M.S.W., M.T., D.D., and S.F.K. contributed the genetic testing and interpretation and review of the manuscript. N.G.C. and R.S.N. were involved in the patient's clinical care and review and editing of the manuscript. 


\section{Funding}

Funding was provided by Grant U19HD077693 from the Eunice Kennedy Shriver National Institute of Child Health and Human Development (NICHD) and the National Human Genome Research Institute (NHGRI).

\section{REFERENCES}

Babenko AP, Polak M, Cavé H, Busiah K, Czernichow P, Scharfmann R, Bryan J, Aguilar-Bryan L, Vaxillaire M, Froguel P. 2006. Activating mutations in the ABCC8 gene in neonatal diabetes mellitus. N Engl J Med 355: 456-466. doi:10.1056/NEJMoa055068

Balboa D, Saarimäki-Vire J, Borshagovski D, Survila M, Lindholm P, Galli E, Eurola S, Ustinov J, Grym H, Huopio $\mathrm{H}$, et al. 2018. Insulin mutations impair $\beta$-cell development in a patient-derived iPSC model of neonatal diabetes. Elife 7: e38519. doi:10.7554/eLife.38519

De Franco E, Flanagan SE, Houghton JAL, Allen HL, Mackay DJG, Temple IK, Ellard S, Hattersley AT. 2015. The effect of early, comprehensive genomic testing on clinical care in neonatal diabetes: an international cohort study. Lancet 386: 957-963. doi:10.1016/S0140-6736(15)60098-8

De León DD, Stanley CA. 2008. Permanent neonatal diabetes mellitus. In GeneReviews ${ }^{\circledR}$ (ed. Adam MP, et al.) pp. 1993-2018. University of Washington, Seattle, WA.

Edghill EL, Flanagan SE, Patch AM, Boustred C, Parrish A, Shields B, Shepherd MH, Hussain K, Kapoor RR, Malecki $\mathrm{M}$, et al. 2008. Insulin mutation screening in 1,044 patients with diabetes: mutations in the INS gene are a common cause of neonatal diabetes but a rare cause of diabetes diagnosed in childhood or adulthood. Diabetes 57: 1034-1042. doi:10.2337/db07-1405

Farnaes L, Hildreth A, Sweeney NM, Clark MM, Chowdhury S, Nahas S, Cakici JA, Benson W, Kaplan RH, Kronick R, et al. 2018. Rapid whole-genome sequencing decreases infant morbidity and cause of hospitalization. NPJ Genom Med 3: 10. doi:10.1038/s41525-018-0049-4

lafusco D, Massa O. 2012. Pasquino B, the early diabetes study group of ISPED minimal incidence of neonatal/ infancy onset diabetes in Italy is 1:90,000 live births. Acta Diabetol 49: 405-408. doi:10.1007/s00592-011 0331-8

Konuş OL, Ozdemir A, Akkaya A, Erbaş G, Celik H, Işik S. 1998. Normal liver, spleen, and kidney dimensions in neonates, infants, and children: evaluation with sonography. AJR Am J Roentgenol 171: 1693-1698. doi:10.2214/ajr.171.6.9843315

Liu M, Hodish I, Haataja L, Lara-Lemus R, Rajpal G, Wright J, Arvan P. 2010. Proinsulin misfolding and diabetes: mutant INS gene-induced diabetes of youth. Trends Endocrinol Metab 21: 652-659. doi:10.1016/j.tem 2010.07.001

Liu M, Sun J, Cui J, Chen W, Guo H, Barbetti F, Arvan P. 2015. INS-gene mutations: from genetics and $\beta$ cell biology to clinical disease. Mol Aspects Med 42: 3-18. doi:10.1016/j.mam.2014.12.001

Oyadomari S, Araki E, Mori M. 2002. Endoplasmic reticulum stress-mediated apoptosis in pancreatic $\beta$-cells. Apoptosis 7: 335-345. doi:10.1023/A:1016175429877

Park JH, Kang JH, Lee KH, Kim NH, Yoo HW, Lee DY, Yoo EG. 2013. Insulin pump therapy in transient neonatal diabetes mellitus. Ann Pediatr Endocrinol Metab 18: 148-151. doi:10.6065/apem.2013.18.3 .148

Pearson ER, Flechtner I, Njølstad PR, Malecki MT, Flanagan SE, Larkin B, Ashcroft FM, Klimes I, Codner E, lotova $\mathrm{V}$, et al. 2006. Switching from insulin to oral sulfonylureas in patients with diabetes due to Kir6.2 mutations. N Engl J Med 355: 467-77. doi:10.1056/NEJMoa061759

Polak M, Cave H. 2007. Neonatal diabetes mellitus: a disease linked to multiple mechanisms. Orphanet J Rare Dis 2: 12. doi:10.1186/1750-1172-2-12

Richards S, Aziz N, Bale S, Bick D, Das S, Gastier-Foster J, Grody WW, Hegde M, Lyon E, Spector E, et al. 2015. ACMG Laboratory Quality Assurance Committee. Standards and guidelines for the interpretation of sequence variants: a joint consensus recommendation of the American College of Medical Genetics and Genomics and the Association for Molecular Pathology. Genet Med 17: 405-424. doi:10.1038/gim .2015 .30

Støy J. 2014. Mutations in the insulin gene [internet]. Diapedia 4105254815 rev. doi:10.14496/dia .4105254815 .6

Suzuki S, Koga M. 2014. Glycemic control indicators in patients with neonatal diabetes mellitus. World J Diabetes 5: 198-208. doi:10.4239/wjd.v5.i2.198

Tubiana-Rufi N. 2007. Insulin pump therapy in neonatal diabetes mellitus. Endocr Dev 12: 67-74. doi:10.1159/ 000109606 
Wirth M, Jellimann JM, Jellimann S, Hascoët JM. 2018. Neonatal diabetes mellitus: improved screening and early management of an underestimated disease. Clin Case Rep 6: 18-22. doi:10.1002/ ccr3.1276

Yang H, Robinson PN, Wang K. 2015. Phenolyzer: phenotype-based prioritization of candidate genes for human diseases. Nat Methods 12: 841-843. doi:10.1038/nmeth.3484

Yildiz M, Akcay T, Aydin B, Akgun A, Dogan BB, De Franco E, Ellard S, Onal H. 2018. Emergence of insulin resistance following empirical glibenclamide therapy: a case report of neonatal diabetes with a recessive INS gene mutation. J Pediatr Endocrinol Metab 31: 345-348. doi:10.1515/jpem-2017-0325 


\section{COLD SPRING HARBOR Molecular Case Studies}

\section{Neonatal diabetes mellitus due to a novel variant in the INS gene}

Sarah E. Laurenzano, Cory McFall, Linda Nguyen, et al.

Cold Spring Harb Mol Case Stud 2019, 5: a004085 originally published online June 13, 2019 Access the most recent version at doi: $10.1101 /$ mcs.a004085

References This article cites 20 articles, 1 of which can be accessed free at: http://molecularcasestudies.cshlp.org/content/5/4/a004085.full.html\#ref-list-1

License This article is distributed under the terms of the Creative Commons Attribution-NonCommercial License, which permits reuse and redistribution, except for commercial purposes, provided that the original author and source are credited.

Email Alerting Receive free email alerts when new articles cite this article - sign up in the box at the Service top right corner of the article or click here. 